Arthroskopie 2014 $\cdot 27: 245-245$

DOI 10.1007/s00142-013-0795-3

Online publiziert: 25 . September 2014

(c) Springer-Verlag Berlin Heidelberg 2014

\section{H. Lill ${ }^{1} \cdot$ K.-H. Frosch ${ }^{2}$}

${ }^{1}$ Klinik für Unfall- und Wiederherstellungschirurgie, Abteilung des Berufsgenossenschaftlichen Unfallkrankenhauses Hamburg, Diakoniekrankenhaus Friederikenstift gGmbH, Hannover

${ }^{2}$ Chirurgisch-Traumatologisches Zentrum, Asklepios Klinik St. Georg, Hamburg

\title{
Arthroskopie bei Frakturen
}

Die Arthroskopie ist mittlerweile fest in der Versorgung intraartikulärer Frakturen etabliert und wird aktuell auch bei extraartikulären Frakturen, z. B. der lateralen Klavikulafraktur, sinnvoll angewandt. Im Fokus steht hierbei nicht nur die Kontrolle des Repositionsergebnisses, sondern vielmehr auch die Erkennung und Therapie intraartikulärer Begleitverletzungen. Ein weiterer wichtiger Effekt stellt die kontrollierte Gelenklavage dar.

Das Komitee TRAUMA der AGA beschäftigt sich seit Jahren mit diesem Thema an unterschiedlichen Lokalisationen. Hierbei wurden sowohl die Möglichkeiten als auch die Grenzen der Arthroskopie analysiert und in diesem Heft als Übersichtsartikel zusammengefasst. Dabei wurde insbesondere Wert auf den aktuellen wissenschaftlichen Hintergrund sowie auch auf die praktische Durchführung mit Tipps und Tricks gelegt. Entstanden sind hervorragende Übersichtsarbeiten, die von Spezialisten auf dem jeweiligen Gebiet verfasst wurden.

Hervorzuheben sind neue arthroskopische Verfahren in der Behandlung der lateralen Klavikulafraktur, der Glenoidfraktur, der proximalen Humerusfraktur und der Patellafraktur, aber auch in der Therapie von Frakturen am Ellenbogen sowie am oberen und unteren Sprunggelenk. Die Arthroskopie bei Tibiakopffrakturen ist die bisher am weitesten verbreitete Indikation. Dabei hat sich in zahlreichen Studien gezeigt, dass die Reposition gleichermaßen exakt bildwandlerkontrolliert durchgeführt werden kann, jedoch Begleitverletzungen, wie z. B. Knorpel- und Meniskusschädigungen, im Vordergrund stehen. Die Arthroskopie bei akuten distalen, intraartikulären Radiusfrakturen hat sich im klinischen Alltag nicht etabliert, wird jedoch bei bestimmten Fragestellungen und begleitenden Kapsel-Band-Verletzungen eingesetzt. Hierbei eröffnen sich interessante therapeutische Aspekte.

Kritisch angemerkt wird immer wieder, dass die arthroskopischen Techniken einen deutlichen Mehraufwand an Material und Zeit bedeuteten. Gerade die gegenüber offenen Verfahren oft eingeschränkte Übersicht, das Arbeiten durch Trokare etc. erfordern entsprechende Erfahrung und Zeit. Die Anforderungen an die Arthroskopeure sind durch die erhebliche Erweiterung des Spektrums deutlich höher. Daraus resultiert, dass arthroskopisch gestützte Verfahren viel Expertise erfordern, auch wenn sie minimal-invasiv sind.

Wir hoffen, dass Ihnen die Übersichtsarbeiten praxisnah neue Wege zeigen und etablierte arthroskopisch gestützte Frakturversorgungen kritisch beleuchten.

Viel Freude beim Lesen!

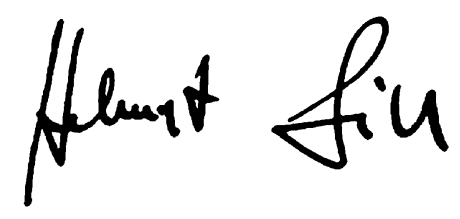

Helmut Lill

Mitglied des Komitees TRAUMA und Generalsekretär der AGA

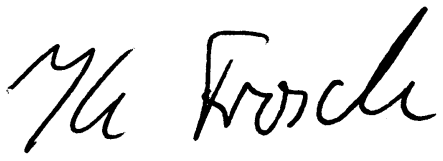

Karl-Heinz Frosch

Vorsitzender des Komitees TRAUMA der AGA

\section{Korrespondenzadressen}

Prof. Dr. H. Lill

Klinik für Unfall- und Wiederherstellungschirurgie, Abteilung des Berufsgenossenschaftlichen Unfallkrankenhauses Hamburg, Diakoniekrankenhaus Friederikenstift gGmbH Humboldtstr. 5, 30169 Hannover helmut.lill@ddh-gruppe.de

\section{Prof. Dr. K.-H. Frosch}

Chirurgisch-Traumatologisches Zentrum, Asklepios Klinik St. Georg

Lohmühlenstrasse 5, 20099 Hamburg

k.frosch@asklepios.com

Interessenkonflikt. H. Lill und K.-H. Frosch geben an, dass kein Interessenkonflikt besteht. 